\title{
Avaliações formativas coordenadas por estratégias de participação inspiradas na abordagem de ensino híbrido
}

\author{
Maria Angélica Figueiredo Oliveira ${ }^{1}$, José Valdeni de Lima ${ }^{1}$, Gisele R. Paim ${ }^{2}$ \\ ${ }^{1}$ Pós-Graduação em Informática na Educação - \\ Centro -Av. Paulo Gama, 110 -Anexo III - $3^{\circ}$ andar - \\ CEP: 90040060 - Universidade Federal do Rio Grande do Sul (UFRGS) \\ Porto Alegre - RS - Brazil. \\ ${ }^{2}$ Instituto Federal de Educação, Ciência e Tecnologia Farroupilha (IFFar) - \\ Campus Júlio de Castilhos \\ RS 527 - Estrada de acesso secundário para Tupanciretã \\ Júlio de Castilhos - RS. \\ mariaangelicafodgmail.com, valdenieinf.ufrgs.br,
gisele.paimeiffarroupilha.edu.br
}

\begin{abstract}
This article presents an experience report that brings the insertion of pedagogical practices, based on the integration of hybrid teaching approaches applied in the chemical discipline of the first year of High School in a technical school. The experiments showed that the group that received the classes mediated by the hybrid approach had better results that influenced the participation and performance of the students, compared to the group that experienced the traditional approach. These data proved that by incorporating different ways of working content in class, allowing the student to be aware of its evolution through immediate feedback, as formative action can be a way to more successful results.
\end{abstract}

Resumo. Este artigo apresenta um relato de experiência que traz a inserção de práticas pedagógicas, baseada na integração de abordagens de ensino hibrido aplicadas na disciplina de química do primeiro ano do Ensino Médio de uma escola técnica. Os experimentos mostraram que o grupo com as aulas mediadas pela abordagem híbrida teve melhores resultados, que influenciaram na participação e desempenho dos estudantes, comparados ao grupo que experienciou a abordagem tradicional. Esses dados comprovaram, que ao incorporar formas diferenciadas de trabalhar o conteúdo em aula, permitir ao estudante a consciência da sua evolução, através de feedbacks imediatos, como ação formativa pode ser um caminho para resultados mais bem-sucedidos.

\section{Introdução}

O processo ensino-aprendizagem de química apresenta muitos conteúdos, sendo comum os alunos apresentarem dificuldades de relacionar a teoria com a prática (DE ALMEIDA et al., 2008). Para Gouveia et al. (2018), a falta de atividades experimentais que envolvam a investigação pode desencadear desinteresse em aula. Nesse sentido, a inserção de dinâmicas diferenciadas em sala de aula é fundamental para que o aluno consiga observar a relevância do conteúdo estudado e, com isso, atribuir sentido ao estudo. No contexto 
VIII Congresso Brasileiro de Informática na Educação (CBIE 2019)

Anais do XXV Workshop de Informática na Escola (WIE 2019)

da presente experiência, a disciplina de química é o componente que mais reprova da área básica, portanto a inserção de novas práticas pode favorecer e contribuir para o desenvolvimento de uma boa compreensão conceitual, auxiliando no seu desempenho.

O relatório do Fórum Econômico Mundial de $2018^{1}$ prevê a aprendizagem ativa como uma das competências fundamentais para futuros profissionais e a escola pode ser o ponto de partida para o desenvolvimento de novas habilidades. A aprendizagem ativa para Bacich e Moran (2018) pode ser potencializada pelo ensino híbrido, pois proporcionará benefícios como flexibilidade, compartilhamento de espaços, tempos, tecnologias, fora e dentro da sala de aula, evidenciando, através de práticas ativas, o protagonismo do aluno, transformando-o em mais participativo em todo processo. Somadas à inserção dessas boas práticas, a avaliação também necessita ser contemplada no processo ensino-aprendizagem. Desse modo, o presente trabalho propôs a integração de diferentes práticas pedagógicas, oportunizando ao estudante transitar por estilos e recursos diferentes, proporcionando uma sala de aula que maximiza o envolvimento de todos, razão pela qual aproxima o professor e aluno, auxiliando, antes das avaliações, a detectar futuros direcionamentos na disciplina, bem como identificar quem necessita de maior auxílio. Também, permite ao aluno a condição de autorregulação, a partir das avaliações formativas no processo ensino-aprendizagem.

\section{Ensino Híbrido}

A aprendizagem híbrida envolve a reunião de elementos da sala de aula tradicional (presencial) com a sala de aula on-line. Bacich et al. (2015) enfatiza que esses elementos beneficiam o trabalho didático com metodologias ativas, pois promovem atividades que incentivam uma postura mais ativa dos estudantes, enriquecendo cada vez mais o processo de aprendizagem. Para Horn e Staker (2015) o ensino híbrido pode ser dividido em quatro grandes modelos: rotação, flex, à la carte e virtual aprimorado. $\mathrm{O}$ enfoque do presente artigo foi no modelo de rotação, mais especificamente no subtipo de rotação por estações e sala de aula invertida. A ideia do modelo de rotação é proporcionar o revezamento de atividades dispostas em estações, predeterminadas pelo professor, sendo uma delas necessariamente em meio virtual. A quantidade de estações e o tempo que o aluno ficará em cada estação irá depender da infraestrutura da escola, carga horária da disciplina e as atividades que serão desenvolvidas. Uma das grandes vantagens da implementação desse modelo é oportunizar diferentes estratégias de participação, tanto individuais ou coletivas, além de produzir um efeito dinâmico em sala de aula.

No que tange ao subtipo sala de aula invertida, a ideia desse conceito é promover a inversão da sala de aula tradicional, oportunizando o acesso e o estudo do conteúdo pelo aluno antes da aula. Bergmann e Sams (2014) acreditam que essa prática qualifica a interação entre estudantes e professores, visto que a as aulas passam ser espaços em que a discussão e a resolução de problemas predominam entre as atividades, dando o lugar a aulas mais participativas e menos expositivas, aproximando o professor das necessidades da turma. O método ativo de ensino Peer Instruction (PI) idealizado por Mazur (1997), embora seja um método que independa de Tecnologias de Informação e Comunicação (TIC) para a sua implementação, pode ser considerado como uma sala de aula invertida, quando as TICs são integradas ao processo ensino-aprendizagem. Um dos pontos centrais

\footnotetext{
${ }^{1}$ WEF. The Future of Jobs Report. World Economic Forum, 2018. Report 2018-2022. Disponível em: http://www.weforum.org. Acesso em 15 de Fevereiro de 2019.
} 
VIII Congresso Brasileiro de Informática na Educação (CBIE 2019)

Anais do XXV Workshop de Informática na Escola (WIE 2019)

do método, segundo Araújo e Mazur (2013), reside no momento do feedback das questões conceituais, a instrução por pares (tradução de peer instruction) ou a discussão somente acontecerá quando o feedback de acertos for entre $30 \%$ a $70 \%$, sendo que os estudantes são incentivados a se reunirem em pequenos grupos para convergirem para uma solução. Feedbacks de acertos menores à faixa percentual de referência, a questão é retomada, caso contrário novas questões são realizadas ou um novo conceito é trabalhado.

Muitas pesquisas, como Oliveira e Rechia (2017), Kielt et al. (2017), Nielsen et al. (2014) demonstraram os benefícios do método em sala de aula. Destaca-se os estudos de Mazur e Watkins (2007) que identificaram na estrutura do método PI uma oportunidade aos alunos desenvolverem habilidades de argumentação, independente do tema estudado e, sobretudo, fortalecer a construção do conhecimento mediante discussão.

\section{Avaliações Formativas}

Tradicionalmente a escola compreende a avaliação como um instrumento escalonador, definindo uma lógica classificatória: aqueles que atingiram ou superaram uma média ou conceito predeterminado ou aqueles que não alcançaram ou ficaram aquém do esperado (DEMO, 2010; FERNANDES, 2014). Esse cenário é tão inerente e enraizado que se propaga até os dias atuais mediante tantas tecnologias e ferramentas acessíveis para a escola. A avaliação, na arquitetura didática, é tida com uma atividade separada do ensino e aprendizagem. Basicamente o que se imprime é uma sequência de ensino e aprendizagem como ações que andam juntas, porém a avaliação é um elemento à parte, conclusivo dessa sequência virtual definida como ensino-aprendizagem-avaliação. Tanto é real como é comum em muitas escolas terem a semana das avaliações, agendada no calendário escolar e esperada por muitos alunos com entusiasmo e por tantos outros com apreensão e desânimo. Para Sanmartí (2009) e Luckesi (2008), as emoções podem gerar muitos obstáculos para aprender, caracterizando-se como uma das principais causas de erros e dificuldades de aprendizagem. O desempenho escolar aliado ao fracasso escolar e às formas de avaliação são uns dos fatores que influenciam nos índices de retenção e reprovação em uma escola (DORE; LÜSCHER, 2013; LUCKESI, 2011).

Para Rabelo (1998, p. 70), qualquer tipo de avaliação pressupõe objetos e critérios, ao contrário do que acontece normalmente nas escolas, onde o único avaliado é o aluno ou, por vezes, somente a sua aprendizagem. No entanto, segundo o autor, no processo ensino-aprendizagem, outras questões deveriam ser avaliadas, como, por exemplo, os objetivos, conteúdos, as propostas de intervenções didáticas e os recursos utilizados. Rabelo (1998) reuniu em sua pesquisa alguns tipos de avaliação que são consenso entre muitos autores (BLOOM; HASTINGS; MADAUS, 1983; SANMARTÍ, 2009), categorizando-as quanto: à regularidade da avaliação, podendo ser pontual ou contínua; quanto ao avaliador, que pode vir a ser interno ou externo; quanto à comparação, que pode ser normativa ou critério; e quanto à formação, que pode ser diagnóstica, formativa e somativa. No que tange a formação, foco deste artigo, Sanmartí (2009) destaca a avaliação formativa como a mais importante para os resultados da aprendizagem, dentro da concepção cognitivista ela possibilita compreender a razão de um aluno não entender um conceito ou não conseguir concretizar uma atividade. Quando realizada ao longo desse processo, é fundamental para o estudante poder identificar suas dificuldades, compreendê-las permitindo assim a autorregulação.

[...]na avaliação tradicional, a regulação da aprendizagem é fundamentalmente realizada pelos professores, já que é a eles delegada a função de detectar as 
VIII Congresso Brasileiro de Informática na Educação (CBIE 2019)

Anais do XXV Workshop de Informática na Escola (WIE 2019)

dificuldades e os acertos dos alunos, analisá-los, e tomar decisões. No entanto, está provado que somente o próprio aluno pode corrigir seus erros, apercebendo-se do por que se equivoca. [...] A função dos professores deveria se centrar, assim em compartilhar com os alunos este processo avaliativo. Não é suficiente que aquele que ensina "corrija" os erros e "explique" a visão correta, deve ser o próprio aluno que se avalia, propondo-se atividades com esse objetivo específico. (SANMARTÍ, 2009, p. 19).

A avaliação formativa contribui para melhorar a aprendizagem em todo estágio de formação, pois informa o professor sobre o desenvolver do seu processo de ensinoaprendizagem do aluno, assim como, para o aluno, a consciência do seu próprio caminhar através de feedbacks rápidos e úteis sobre todo o processo, assumindo uma função reguladora quando possibilita que alunos e professores adaptem ou ajustem suas estratégias e dispositivos (RABELO,1998). Bloom, Hastings e Madaus (1983) identificam como a maior qualidade da avaliação formativa o auxílio ao aluno em relação à sua aprendizagem e a ditar o ritmo dela, mudando a postura do aluno, que muitas vezes tende a adiar o estudo por falta de tempo ou em razão de um número grande de disciplinas, sendo que o próprio estudante acaba não elegendo como prioridade as áreas com solicitações menos urgentes. A avaliação formativa possibilita um maior engajamento em aula ao passo que as avaliações e feedbacks são constantes e parte do processo. As TICs podem potencializar o feedback em aula, tornando o processo mais ágil, pois facilita ao professor a identificação imediata das respostas dos alunos em tempo real. Schiehl et al. (2017) corrobora, afirmando em seus estudos que a avaliação formativa é a melhor forma de promover um desempenho mais eficaz, sobretudo em contextos de ensino híbrido.

Além do uso de Ambientes Virtuais de Aprendizagem (AVA), que fornecem recursos síncronos e assíncronos, existe uma diversidade de ferramentas, muitas delas gratuitas, que podem ser inseridas na organização didática com a finalidade de promover avaliações formativas por meio de dispositivos eletrônicos, transformando a sala de aula em um espaço híbrido, resultando em uma experiência mais participativa.

Uma dessas ferramentas é a Socrative ${ }^{2}$, definida como uma plataforma on-line disponibilizada de forma gratuita, sendo acessível em dispositivos móveis ou computador pessoal, proporcionando aos professores e alunos um espaço interativo com suporte para 50 alunos conectados por sessão. A ferramenta disponibiliza diferentes atividades em formato de perguntas, espaço para competição entre os estudantes, além de relatório de acompanhamento em tempo real ao professor, auxiliando a direcionar a aprendizagem conforme a análise das respostas. Existem alguns trabalhos, como de Basso e Loyer (2016), Benítez-Porres (2015), Vettori e Zaro (2016), que adotaram a ferramenta socrative em práticas pedagógicas e os resultados demonstram um aumento de interação em sala de aula, engajamento dos estudantes, além de ser um recurso que não dispende nenhum custo, provocando uma experiência transformadora de aprendizagem.

Outra ferramenta é a Kahoot! (C) ${ }^{3}$, uma plataforma on-line de aprendizagem baseada em jogos, disponível gratuitamente. Possui cadastro para professores em que permite organizar as tarefas por tópicos e disponibilizá-las em formato de quiz, discussões e lacunas de conceitos. Também fornece ao professor a possibilidade de definir tempo para contagem regressiva das respostas, progresso dos estudantes, feedback das respostas, gráfico com a quantidade de alunos e respostas em tempo real com os acertos e erros. A

\footnotetext{
${ }^{2} \mathrm{http}: / /$ www.socrative.com

${ }^{3}$ http://www.kahoot.com
} 
VIII Congresso Brasileiro de Informática na Educação (CBIE 2019)

Anais do XXV Workshop de Informática na Escola (WIE 2019)

ferramenta também disponibiliza o tempo de resposta de cada estudante ou da equipe, que podem ser salvos no computador ou "na nuvem" no formato de planilha eletrônica e, também, fornece placar e ranking dos cinco melhores desempenhos (IZEKI, 2016). Várias pesquisas demonstram resultados positivos, em sala de aula, com a inserção da ferramenta Kahoot!. Alguns exemplos de sucesso podem ser vistos nos trabalhos desenvolvidos por Da Costa et al. (2017), Gazotti-Vallim et al. (2017) e Zarzycka-Piskorz (2016). Esses autores constataram uma melhoria na aprendizagem, mediada pela construção e visualização de conceitos, além de promover o envolvimento dos estudantes ao participarem de uma atividade gamificada.

A ferramenta Poll Everywhere ${ }^{4}$ é outra opção, considerada uma plataforma online, economicamente acessível, fácil de usar e que pode melhorar a aprendizagem dos alunos e a experiência da sala de aula (SHON e SMITH, 2011). A ferramenta possibilita ao professor a criação de questionários que podem ser facilmente respondidos de qualquer dispositivo, sendo que as respostas às questões podem ser de múltipla escolha, abertas ou através de imagens e questões que os alunos podem fazer ao professor. A interação também pode ocorrer por mensagens de texto ao celular do professor e por Twitter. Para acesso como aluno, o professor compartilha o poll (votação), onde é gerada uma identificação em formato de link para o estudante participar. A ferramenta suporta até 40 alunos conectados, mas esse número pode ser maximizado pelo acesso do Twitter ou mensagens de texto.

Plickers ${ }^{5}$ é outro recurso educacional que pode potencializar o processo de avaliação formativa. Por ser uma ferramenta on-line e gratuita de simples operação, possibilita aos professores, por meio do seu celular, a construção e coleta de dados de avaliações formativas realizadas em tempo real sem a necessidade do uso de dispositivos móveis pelos estudantes, visto que o recurso gera cartões identificados por um $Q R$ code, que devem ser impressos e entregues para cada aluno, os quais são facilmente reconhecidos por leitores de celulares com câmera integrada (BESSA e NUNES, 2017). Os mesmos autores ainda citam a dinamicidade que a ferramenta promove ao mensurar de forma imediata, o nível de aprendizado do aluno e direcionar, por meio de relatórios gerados ao final do ciclo de respostas de cada questão, uma revisão ou reforço sobre o conteúdo aos que obtiveram um baixo rendimento (2017, p. 734).

\section{Metodologia}

A experiência realizada e apresentada neste artigo envolveu a participação de 57 estudantes do primeiro ano do Ensino Médio de uma escola técnica. Os experimentos foram aplicados na disciplina de química. Os estudantes foram divididos em dois grupos classificados como experimental e controle, conforme figura 1 . O conteúdo da disciplina abordado foi sobre Isótopos, Isóbaros e Isótonos. Para ambos grupos o material foi disponibilizado no ambiente virtual de aprendizagem, sendo utilizados vídeos de apoio sobre o conceito trabalhado. A dinâmicas das aulas do grupo experimental seguiu a estrutura da abordagem híbrida (sala de aula invertida e rotação por estações) e, a do grupo de controle recebeu a abordagem tradicional (baseada na exposição do conteúdo).

\footnotetext{
${ }^{4} \mathrm{http}: / /$ www.polleverywhere.com

${ }^{5} \mathrm{http}: / / \mathrm{www}$.plickers.com
} 
VIII Congresso Brasileiro de Informática na Educação (CBIE 2019)

Anais do XXV Workshop de Informática na Escola (WIE 2019)

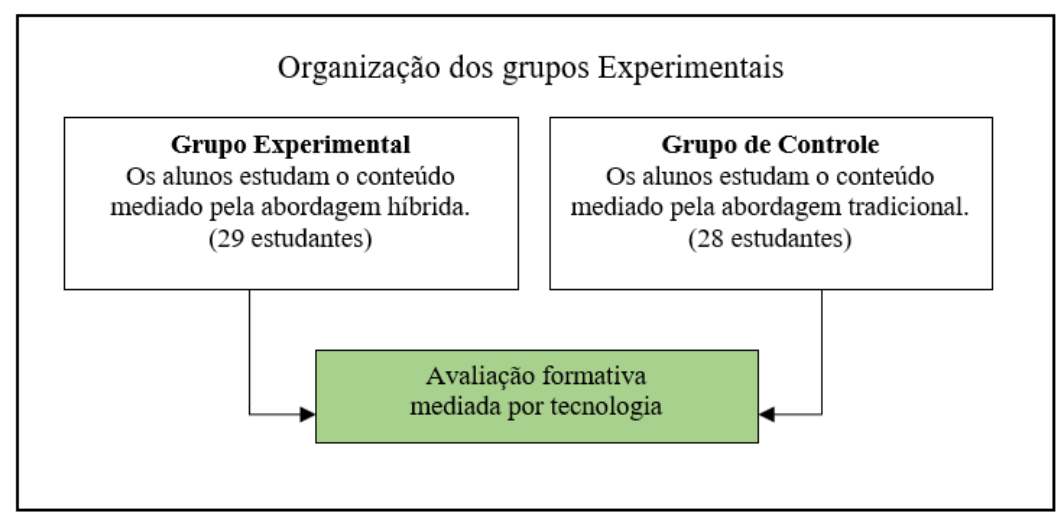

Figura 1. Organização dos experimentos.

Em ambos grupos, as aulas durante o período do experimento, foram realizadas no laboratório de informática. No que se refere as aulas com a abordagem híbrida, foram definidas duas estações de atividades, com 15 minutos cada, sendo uma estação de estudo individual, onde os alunos estudavam o conteúdo em vídeo disponibilizado no ambiente virtual da escola e, a outra estação denominada colaborativa, os alunos estudavam em pares sobre o mesmo conteúdo, fazendo uso do material didático impresso da disciplina. Em ambos grupos houve a integração das avaliações formativas mediadas pela ferramenta Kahoot!, escolhida pela facilidade de utilização e por oferecer um ambiente gamificado à essa atividade. As avaliações formativas foram compostas de questões objetivas com quatro alternativas, respondidas individualmente e registradas na ferramenta. Nesse momento a dinâmica das avaliações foram inspiradas no método PI, oportunizando aos estudantes um espaço para discussões decorrentes da divergência que eventualmente fossem geradas nas respostas. Para medir a evolução dos dois grupos foram realizados pré-testes antes de iniciar os experimentos e pós-testes ao final, contendo cinco questões objetivas sobre o conteúdo, aplicados em ambos grupos. As mesmas questões foram aplicadas para o pré-teste e pós-teste, visto que o período de intervalo de um teste para outro correspondeu a quatro semanas. Na próxima seção são detalhadas as discussões e resultados decorrentes da execução dos experimentos.

\section{Discussões e Resultados}

Nesta seção, são apresentados os experimentos realizados durante as três semanas de execução em aula, assim como as discussões e resultados coletados em cada grupo. Os experimentos planejados, neste trabalho, tiveram como intuito de verificar os efeitos de cada prática no desempenho dos estudantes, assim como na dinâmica das aulas neste período.

Constatou-se, conforme tabela 1, que ambos grupos partiram do mesmo grau de conhecimento em relação ao conteúdo, no entanto é no pós-teste que é apresentada a maior diferença. Identificou-se com o pós-teste uma evolução do grupo experimental, representando uma diferença acentuada para o grupo de controle, o que permite inferir que a abordagem híbrida teve um papel diferenciado no desempenho. Essa constatação é confirmada pelo valor de $\mathrm{p}<0,05$, nível de significância adotado, a partir da aplicação do teste de Mann-Whitney para mensurar a média dos dois grupos independentes (controle e experimental). O teste mostrou que houve um ganho de desempenho na turma experimental, ratificado pela diferença significativa entre as médias dos grupos e pela variância obtida. Essa variância revela que a dispersão das notas em relação à média é 
VIII Congresso Brasileiro de Informática na Educação (CBIE 2019)

Anais do XXV Workshop de Informática na Escola (WIE 2019)

maior no grupo de controle e com uma amplitude menor no grupo com as aulas medidas pela abordagem híbrida.

Tabela 1. Desempenho dos estudantes nos experimentos de química

\begin{tabular}{|c|c|c|}
\hline & Controle & Experimental \\
\hline Média (pré-teste) & 3,64 & 3,66 \\
\hline Média (pós-teste) & 4,14 & 6,07 \\
\hline Variância (pós-teste) & 8,87 & 4,71 \\
\hline $\begin{array}{c}\text { Teste Mann-Whitney } \\
\text { p-valor }\end{array}$ & \multicolumn{2}{|c|}{0,014} \\
\hline
\end{tabular}

A quantidade de avaliações formativas variou entre os grupos, visto que o tempo de aprendizagem ativa foi mais eficaz na turma experimental, proporcionado uma estratégia de participação mais ativa entre os alunos, ao contrário do grupo de controle em que o conteúdo foi abordado de forma muito mais expositiva e, desse modo, apresentando uma participação mais limitada.

Percebeu-se que a potencialização da participação no grupo experimental foi decorrente da diversidade de atividades realizadas, resultando em uma inatividade menor em sala de aula, visto que a integração das estações de maneira combinada com os momentos de avaliação formativa despertou uma maior interesse e envolvimento dos alunos com o conteúdo. Entende-se que esse tempo de estudo caracterizado pela aprendizagem ativa e reativado com a transição entre as estações foi o diferencial para o desempenho alcançado pelo grupo experimental.

Outro aspecto importante, comum em ambos grupos, evidenciam a importância que tem ofeedback imediato proveniente das avaliações formativas, fornecendo subsídios que permitem ao professor acompanhar a evolução da turma e traçar direcionamentos da disciplina e, consequentemente, ao estudante possibilita ter o controle do seu próprio progresso. Ressalta-se que a ferramenta kahoot potencializou o processo de feedback por fornecer a porcentagem geral de acertos e erros, além da análise individual do desempenho (assertibilidade e tempo) de cada estudante.

Observou-se com esse experimento que, quanto mais participativo o estudante for em sala de aula mais preparado ele estará para as discussões e consequentemente para as avaliações formativas. As discussões, inspiradas no método PI, foram mais produtivas no grupo experimental devido ao fator participação ter sido maior, sobretudo pelo momento de estudo nas estações, o que contribuiu de maneira produtiva para a aprendizagem, refletindo significativamente na melhoria do desempenho.

\footnotetext{
${ }^{6}$ Mann-Whitney é um teste não paramétrico utilizado em planejamentos experimentais do tipo aleatório (RODRIGUES, 1975).
} 
No que se refere à avaliação da satisfação pelos estudantes, foi entregue um questionário em que se perguntou a opinião deles em relação as abordagens experenciadas nas últimas aulas. Foi utilizada uma escala likert de cinco pontos, que variava de péssimo a ótimo, em que foi possível medir o grau de satisfação percebido. Os alunos do grupo experimental avaliaram $100 \%$ positivamente as aulas mediadas pela abordagem híbrida, variando antes as opções ótimo $(85 \%)$ e bom (15\%). Em contraste, no grupo de controle as opiniões dos estudantes divergiram, mostrando um percentual menor, com $48 \%$ das avaliações positivas.

Em ambos grupos, o momento das avaliações formativas mediadas pela ferramenta kahoot! foi a atividade preferida pelos estudantes, fortalecida no grupo experimental pelo estudo nas estações, o que o tornou as aulas mais dinâmicas. Percebeuse que o envolvimento ativo do estudante influencia diretamente na sua aprendizagem, sendo comprovado pelos resultados mais bem-sucedidos do grupo experimental.

\section{Considerações Finais}

$\mathrm{O}$ artigo apresentou uma experiência realizada na disciplina de química do primeiro ano do Ensino Médio de uma escola técnica. O propósito dessa experiência foi oportunizar aos estudantes práticas diferenciadas na disciplina de modo a envolvê-los de forma ativa na sua aprendizagem, potencializando seu desempenho e, transformando o exercício tradicional da exposição, em uma experiência mais participativa em classe

Possibilitar ao aluno a consciência da sua evolução, por meio de feedbacks imediatos, como ação formativa, pode conduzir a resultados mais bem-sucedidos. No entanto, essa ação se tornará completa quando o aluno se envolver ativamente na sua aprendizagem. Por esse motivo, a abordagem híbrida, distribuída por atividades em estações, inserida nesta experiência, permitiu conhecer e atender melhor às lacunas dos estudantes, como também auxiliou na prática pedagógica a incorporar forma inovadoras de trabalhar o conteúdo em aula.

Tendo em vista os resultados demonstrados no desempenho dos alunos, influenciados por uma postura mais ativa em sala de aula, pretende-se ampliar a utilização dessa prática na disciplina, investigando os seus efeitos com o uso de outros recursos educacionais e com isso agregar e promover experiências inovadoras de construção do conhecimento por meio de uma aprendizagem mais participativa em sala de aula.

\section{Referências}

Araújo, I. S., Mazur, E. (2013) "Instrução pelos Colegas e Ensino sob Medida: uma proposta para o engajamento dos alunos no processo de ensino-aprendizagem de Física”. Caderno Brasileiro de Ensino de Física, v. 30, n. 2: p. 362-384.

Bacich, L.; Moran, J. (2018) Metodologias ativas para uma educação inovadora: uma abordagem teórico-prática. Porto Alegre: Penso.

Bacich, Lilian; Neto, Adolfo Tanzi e TREVISANI, Fernando De Mello. (2015) Ensino híbrido - personalização e tecnologia da educação. Editora Penso: Porto Alegre.

Basso, M. \& Loyer, S. (2016). "Uso de Socrative como elemento de interacción en cursos de Ingeniería”. XXIX Congreso Chileno de Educación en Ingeniería (SOCHEDI), Pucón. 
VIII Congresso Brasileiro de Informática na Educação (CBIE 2019)

Anais do XXV Workshop de Informática na Escola (WIE 2019)

Benítez-Porres, Javier. (2015) "Socrative como herramienta para la integración de contenidos en la asignatura". Didáctica de los Deportes.

Bergmann, J. \& Sams, A. (2014). Flipped learning Gateway to Student Engagement, In: DOMÍNGUEZ, Adelaida Delgado. "Using mobile applications and online services to collect and compute statistics of peer assessments." Information Systems and Technologies (CISTI), 2013 8th Iberian Conference on. IEEE, 2013.Learning \& Learning with Technology, May 18-23.

Bessa, R.C; Nunes. V.W.D.N. (2017). "Uso do aplicativo Plickers como recurso de Metodologia Ativa". II Congresso sobre Tecnologias na Educação (Ctrl+E 2017). Universidade Federal da Paraíba - Campus IV. Mamanguape - Paraíba - Brasil.

Bloom, B.; Hastings, J. T.; Madaus, G. F. (1983) Manual de avaliação formativa e somativa do aprendizado escolar. São Paulo: Pioneira.

Da Costa, Carlos Helaidio Chaves et al. (2017) "Marvinsketch e kahoot como ferramentas no ensino de isomeria". HOLOS, v. 1, p. 31-43.

De Almeida, Elba Cristina S. et al. (2008) "Contextualização do ensino de química: motivando alunos de ensino médio". XVI Encontro Nacional de Ensino de Química (XVI ENEQ) e X Encontro de Educação Química da Bahia (X EDUQUI), Salvador, BA, Brasil-17 a, v. 20.

Demo, P. (2010) Mitologias da avaliação de como ignorar, em vez de enfrentar problemas. 3. ed. Campinas, SP: Autores Associados.

Dore, Rosemary; Lüscher, Ana Zuleima. (2013) "Permanência e evasão na educação técnica de nível médio em Minas Gerais". Cadernos de Pesquisa, v. 41, n. 144, p. 772789.

Fernandes, C. de O. (2014) Avaliação das Aprendizagens sua relação com o papel social da escola. São Paulo: Cortez.

Gazotti-Vallim, Maria Aparecida; GOMES, Silvia Trentin; FISCHER, Cynthia Regina. (2017) "Vivenciando inglês com Kahoot". The ESPecialist, v. 38.

Gouveia, João Victor Vieira Silva et al. (2018) "Correlacionando a teoria com a prática usando experimentação no ensino de química”. Ciclo Revista, v. 3, n. 1.

Horn, M. B.; Staker, H. (2015) Blended: Usando a Inovação Disruptiva para Aprimorar a Educação. Porto Alegre: Penso.

Izeki, C. A.; N., Walter A.; Dias, R. M. C. (2016) "Experiência no Uso de Ferramentas Online Gamificadas na Introdução à Programação de Computadores". Anais do XXII Workshop de Informática na Escola (WIE 2016), p. 301-310.

Kahoot. Make Learning Awesome. Disponível em: <https://www.kahoot.com/>. Acesso em: 20/06/2019.

Kielt, Everton Donizetti; Da Silva, Sani de Carvalho Rutz; MIQUELIN, Awdry Feisser. (2017) "Implementação de um aplicativo para smartphones como sistema de votação em aulas de Física com Peer Instruction”. Revista Brasileira de Ensino de Física, vol. $39, \mathrm{n}^{\circ} 4$, e4405.

Luckesi, Cipriano C. (2011) Avaliação da Aprendizagem componente do ato pedagógico. $1^{\circ}$ ed. São Paulo: Cortez. 
VIII Congresso Brasileiro de Informática na Educação (CBIE 2019)

Anais do XXV Workshop de Informática na Escola (WIE 2019)

. (2008) Avaliação da Aprendizagem Escolar: estudos e proposições. 19.ed.-São Paulo: Cortez.

Mazur e Watkins (2007). “Justin-in- time teaching and Peer Instruction”. Phisycs,pp. 3962, 2007.

Mazur, E. (1997) Peer instruction: A user's manual. Pap/Dskt ed. [S.1.] Prentice Hall, Inc., p. 253.

Nielsen, K.L; Hansen G.; Stav, J.B. (2014) "How the initial thinking period affects student argumentation during peer instruction: students' experiences versus observations". Stud High Educ, 3, 1-15.

Oliveira, M.A.F.; Rechia, M. (2017) "Potencializando o Ensino da lógica com uso de dispositivos móveis mediados pelo método Peer Instruction". Congresso Brasileiro de Informática da Educação (CBIE). XXIII Workshop de Informática Na Escola (WIE).

Rabelo, E. H. (1998) Avaliação: novos tempos, novas práticas. 2.ed. Petrópolis, RJ: Vozes.

Rodrigues, A. (1975) A pesquisa Experimental em Psicologia e Educação. Petrópolis: Editora Vozes.

Sanmartí, N. (2009) Avaliar para Aprender. Tradução Carlos H. L. Lima, Porto Alegre: Artmed.

Schiehl, Edson Pedro; Kemczinski, Avanilde; Gasparini, Isabela. (2017) "As Perspectivas de Avaliar o Estudante no Ensino Híbrido”. RENOTE, v. 15, n. 2.

Shon, Herb; Smith, Laurie. (2011) "A review of Poll Everywhere audience response system". Journal of Technology in Human Services, v. 29, n. 3, p. 236-245.

Vettori, M., Zaro, M.A. (2016) “Avaliação do Socrative App como ferramenta auxiliar de ensino para a construção de aprendizagens significativas em uma disciplina de física geral a partir do Peer Instruction”. V Congresso Brasileiro de Informática na Educação (CBIE 2016). Anais do XXVII Simpósio Brasileiro de Informática na Educação (SBIE 2016), 190-199.

Zarzycka-Piskorz, Ewa; Podchorążych, U1. (2016) "Kahoot it or not? Can games be motivating in learning grammar?" Teaching English with Technology, v. 16, n. 3, p. 17-36. 